\title{
71 Endurance training and temperature regulation
}

Physical performance is always associated with increased heat generation. This is because the chemical energy of nutrients can only be converted into work output at an efficiency rate of around $40 \%$ ( $\triangleright$ Chapter 6 ). Since our survival depends on us living within a temperature-controlled range, any excess heat generated must immediately be channeled off. During strenuous physical exertion or at high ambient temperatures, radiation or convection play only minor roles. Much more important for this process are the dilated vessels and sweat production followed by evaporative cooling. Under these circumstances, the latter constitutes up to $70 \%$ of our heat dissipation. We can produce from 1-2 liters of sweat per hour, in extreme situations as much as 2.5 liters. Approximately half of the sweat produced evaporates off, extracting heat from the body in the magnitude of $575 \mathrm{kcal}$ per liter in dry to moderately humid air. For example, the energy expenditure of a person weighing $70 \mathrm{~kg}$ during a one-hour run over $10 \mathrm{~km}$ is approximately $700 \mathrm{kcal}$, meaning that approx. $420 \mathrm{kcal}$ fall to heat development. On a sunny day between spring and fall, onethird of this amount of energy is added in the form of absorbed sunlight, making it necessary to dissipate nearly $575 \mathrm{kcal}$ of heat in this scenario. That equates to the evaporative cooling of around one liter of sweat.

The body can tolerate minor losses of fluids without problems. However, under intensive exertion, when sweat production is very high, one of the measurable effects of this is a reduction in the circulating blood volume. That causes the heart beat volume to drop, the body to initiate compensatory reactions with an increased heart rate. To achieve optimal thermoregulation, i.e., transfer heat from inside to outside the body, the vessels of the skin show a reflex response called vasodilation, wherein the effective blood volume is lowered even further. Before these various mechanisms cause heat damage, the body's performance capability is initially reduced. This becomes noticeable even if the reduction in flowing blood volume is only around $3 \%$, or approximately $150-180 \mathrm{ml}$.
As a rule of thumb for calculating the effect of overall fluid loss: performance losses of $10 \%$ can be expected for every $1 \%$ of body weight fluid lost.

When compensating for lost water volume, consider the fact that around $40 \%$ of the water volume excreted through the sweat over one hour of endurance training derives from metabolic processes and therefore does not need to be replenished immediately. Therefore, it is sufficient to drink half to one liter of fluids at the most per hour of normal-intensity endurance training at outdoor temperatures. If the body is pumped with too much fluid, there is a short term elevation in blood volume and, as a consequence, a relative fall in electrolyte levels. The thereby lowered salt concentrations caused by the very high-volume fluid overload can induce states of confusion or seizures. 